\title{
Lingua e spazio urbano a Roma nel racconto di Ettore Scola. Il caso di Una giornata particolare (1977)
}

\author{
Antonio Catolfi (Università per Stranieri di Perugia, Italia) \\ Marco Gargiulo (Università di Bergen, Norvegia)*
}

\begin{abstract}
This article aims to focus on two main aspects of Rome urban space vision and representation through Ettore Scola's filmography: on the one hand, we try to decode the interconnections between languages and cinematic architectural space and, on the other hand, we intend to disclose how Scola meant to create a connection between his personal cinematic narrative and the tangled urban space in the city of Rome.

Our investigation is mainly focused on the so called "urban village" Palazzo Federici, a town within the city, which is the A Special Day and The Story of a Poor Young Man's main location.

Palazzo Federici is an architectural complex of 400 dwellings designed by architect Mario De Renzi and built between 1931 and 1937; it is an ideal place to describe a hive shape building, with a squared structure inspired to a small fortified town, with a central courtyard and an empty fountain, that can represent the different faces of the suffocating fascist regime. The interrelation between the social and the architectural structures and between the mental and urban space anatomies are evident in these two films.

Palazzo Federici is a protagonist in the story narrated during the visit of Hitler in Rome, the 6th May 1938, the Special Day when Antonietta (Sophia Loren) and Gabriele (Marcello Mastroianni) meet, and it leads the characters as a dark set for the Story of a Poor Young Man where it describes the drama of human solitude and desperation in a labyrinthine urban environment in Rome.
\end{abstract}

Keywords: multilingualism, film, Scola, architecture, urban space

\section{Introduzione: La Roma di Ettore Scola}

Questo contributo si inserisce in un più ampio progetto di ricerca che vuole analizzare la relazione tra la narrazione filmica e il ruolo dello spazio urbano della città di Roma nei film di Ettore Scola.

Quando parliamo di spazio urbano, intendiamo riferirci a un concetto complesso di spazio in cui sono contemplati anche gli usi linguistici e i comportamenti sociali. Il nostro obiettivo è quello di individuare le strutture che sorreggono l'architettura come spazio vissuto, da un punto di vista geografico e sociale, e l'architettura del sistema sociolinguistico.

${ }^{*}$ I due autori hanno pensato, discusso e redatto insieme l'intero saggio. In particolare Antonio Catolfi ha scritto i paragrafi 1 e 2, Marco Gargiulo i paragrafi 3 e 4. 
Il rapporto tra cinema e architettura non può essere definito solamente come «un problema di progettazione o adattamento dello spazio profilmico», non lo si può ridurre quindi a un mero problema scenografico. È qualcosa di più. L'architettura progetta e predispone i «percorsi dello sguardo» spettatoriale e la regia cinematografica è come l'architettura, programma e dirige l'attenzione degli spettatori in modo da generare emozioni visive (Costa 2002: 97).

Come afferma Giuliana Bruno in Atlante delle emozioni (2015), sia il cinema che l'architettura descrivono lo spazio urbano della città perché sono strettamente legati al paesaggio cittadino, ovvero

attengono [fortemente] allo spazio. [...] il cinema si definisce come pratica spaziale. È una forma d'arte di strada, un fattore della costruzione delle vedute cittadine. Il paesaggio della città finisce per interagire da vicino con le raffigurazioni cinematografiche, pertanto l'edificio urbano è una "costruzione filmica" non meno che architettonica. [...] Il senso del luogo è attivamente prodotto da una costellazione di immaginari, tra cui i film, sia quelli girati in esterni sia quelli che si fabbricano la propria mise en scène» (Bruno 2015: 43).

Roma è molto presente nei film di Ettore Scola. Nonostante fosse irpino di nascita ${ }^{1}$, il regista ha sempre considerato Roma come la sua città, la città in cui si sentiva a casa, e dove ha vissuto per quasi tutta la vita. Come ha ricordato Giuliano Montaldo, Scola ha saputo raccontare Roma come forse nessuno aveva fatto in precedenza, in modo «ironico e pungente» attraverso soprattutto la descrizione dei luoghi e delle persone che si muovevano dentro gli spazi urbani; Roma era «un set a cielo aperto pieno di attori che erano i residenti» (Dionisi, De Pascalis 2016: 276).

Quando Scola ha descritto la città eterna nei suoi film, le ambientazioni non sono state casuali o di facciata, bensì sempre funzionali alle storie che avrebbe voluto raccontare. Difatti, far apparire Roma nei suoi film non è stato un fatto secondario, per motivi produttivi o personali: lo spazio urbano di Roma, in cui è compreso anche lo spazio linguistico, è diventato fondamentale per il racconto, e ciò è vero soprattutto in alcuni film.

Nella produzione di lungometraggi di ambientazione storica, molto spesso si ricostruiscono i luoghi o si scelgono le location in base alla psicologia dei personaggi, che entra dunque a far parte dell'architettura. Questo in Scola è avvenuto quasi sempre, è stato un elemento centrale del suo lavoro di preparazione. La sceneggiatura, infatti, era il fulcro del film; regnava maestra nell'ambito produttivo di ogni suo film, perché Scola nasce prima come grande sceneggiatore, insieme a Ruggero Maccari, a registi come Mario Mattoli e Camillo Mastrocinque $^{2}$, Steno ${ }^{3}$ e soprattutto insieme a Antonio Pietrangeli ${ }^{4}$, Dino Risi ${ }^{5}$. Diventa poi regista dal 1964 con Se permettete parliamo di donne con Vittorio Gassman (Dionisi, De Pascalis 2016: 274-277).

\footnotetext{
${ }^{1}$ Ettore Scola nasce a Trevico, in provincia di Avellino, il 10 maggio 1931.

${ }^{2}$ Mario Mattoli con Totòtarzan (1951), Due notti con Cleopatra (1953), I giorni più belli (1956), Guardatele, ma non toccatele (1959), Non perdiamo la testa (1959); Camillo Mastrocinque con Totò Peppino e... la malafemmina (1956, non accreditato).

${ }^{3}$ Un americano a Roma (1954), Totò nella luna (1958).

${ }^{4}$ Amori di mezzo secolo (1953, Girandola 1910), Lo scapolo (1955), Nata di marzo (1957), Adua e le compagne (1960), Fantasmi a Roma (1961), La parmigiana (1963), Il magnifico cornuto (1964), La visita (1964), Io la conoscevo bene (1965).

5 Il mattatore (1959), Il sorpasso (1962), La marcia su Roma (1962), I mostri (1963), Il gaucho (1964), I complessi (1965, episodio Una giornata decisiva), Il profeta (1968), Noi donne siamo fatte così (1971).
} 
L'importanza che Scola è riuscito a dare all'aspetto urbano dei suoi film ambientati a Roma è testimoniata anche dalla scelta dei suoi collaboratori lungo il corso di tutta la sua carriera.

La produzione di un film, come sappiamo, è la somma di molti linguaggi tecnici: sceneggiatura, fotografia, musica, costumi, scenografia, montaggio, rappresentati da specialisti delle varie arti. Si tratta di professionisti che, esperti nei rispettivi campi d'interesse, aiutano il regista nella realizzazione del film. Ettore Scola ha avuto la tendenza a contrarre un vero e proprio matrimonio professionale con alcuni di loro (Dionisi, De Pascalis 2016: 277). Guardando alla sua produzione complessiva - ben 49 tra film, cortometraggi, documentari e spettacoli teatrali ${ }^{6}$ - troviamo che tra i suoi principali e frequenti collaboratori vi sono proprio coloro che si occupano dell'architettura e del movimento del film: lo scenografo Luciano Ricceri, 24 film insieme, l'arredatore Ezio Di Monte, 14 film, e il montatore Raimondo Crociani, 20 film $^{7}$. A questo va aggiunto, per quanto riguarda Roma, il fatto che Scola, sia in qualità di sceneggiatore sia come regista, ha scritto molti film per i due attori romani più rappresentativi dell'epoca: Alberto Sordi (19 film) e Nino Manfredi (14 film $)^{8}$.

Inoltre, Scola ha scelto, in alcuni film ambientati a Roma, un unico luogo principale dove far muovere e interagire gli attori. Per certi versi, potremmo definire il suo cinema un "cinema teatrale" che ha bisogno di spazi urbani limitati per definire meglio le emozioni. In queste scelte, la sua formazione giovanile da grande estimatore del teatro ha avuto un grande peso, pertanto la scenografia e i luoghi in cui vengono effettuate le riprese diventano fondamentali soprattutto se sono ben determinati e unici. Non importa se reali o se perfettamente ricostruiti.

Vediamo ora alcuni esempi concreti e che sono oggetto di questo contributo. Nei due film Una giornata particolare (1977) e Il Romanzo di un giovane povero (1995), l'ambientazione principale è collocata in un grande palazzo costruito durante il fascismo, Palazzo Federici.

In Brutti sporchi e cattivi (1976), le baracche sono ricostruite su un monte che domina la città e che rivolge la vista verso la basilica di San Pietro: Monte Ciocci, vicino alla stazione di Valle Aurelia9. Una presenza opprimente quella della cupola di S. Pietro, che rappresentava per Scola il simbolo di una vera "assenza", quella della Chiesa Cattolica nei fatti della città, come era già successo nei confronti degli ebrei durante la seconda guerra mondiale (ASC incontri, A. VII n.2, intervista a Scola, p. 22).

In altri due film, Concorrenza sleale (2001) e La famiglia (1987), diventano protagoniste le vie e le case di Roma del quartiere Prati. Questa volta la location è ricreata in

\footnotetext{
${ }^{6}$ A cui vanno aggiunte 58 sceneggiature, 56 in 17 anni di attività come sceneggiatore dal 1951 al 1968.

${ }^{7}$ Uno dei principali collaboratori di Ettore Scola è stato il maestro Armando Trovajoli, buona parte dei suoi film (24), sono stati musicati dal maestro Trovajoli.

${ }^{8}$ Nino Manfredi, nato a Castro dei Volsci in Ciociaria e poi trasferitosi a Roma da bambino, è sempre stato considerato uno degli attori romani per eccellenza. Per completezza, inoltre, va indicato che Scola scrisse sedici film in cui era interprete Vittorio Gassman e dieci film in cui era interprete Marcello Mastroianni.

${ }^{9}$ L'ingresso del Parco di Monte Ciocci, dopo un lungo periodo di degrado e di sistemazione, è ora situato all'incrocio tra via Anastasio II e via Pietro De Cristofaro, poco prima del ponte ferroviario e della stazione metro di Valle Aurelia. Altro ingresso del Parco è alla fine del quartiere Balduina, in Via Lucio Apuleio n. 33. Salendo sul monte si domina la città e la vista della cupola di San Pietro è molto evidente data la vicinanza. Alla fine di Via Lucio Apuleio, in uno degli ingressi del parco, sono presenti delle ex catapecchie ora ristrutturate, questo a testimonianza del fatto che quando Scola decise di girare il film in quel parco era erano realmente presenti delle baracche.
} 
studio, perché dal punto di vista produttivo la ricostruzione era più funzionale. In altri film, come C'eravamo tanto amati (1974) e Gente di Roma (2003), sono invece protagoniste le vie e le piazze del centro, la Fontana di Trevi, il lungotevere, Via del Tritone, Via dei Due Macelli, Piazza del Popolo, ma anche zone non centrali come il quartiere Trieste, per seguire una sorta di abitudine e una contiguità spaziale. Scola, infatti, per molti anni ha abitato in Via Bertoloni, nella parte nord di Roma, in una zona non distante dal quartiere Trieste, location di molti suoi film. Inoltre, va tenuto conto che anche Ruggero Maccari abitava nella parte nord di Roma, nel quartiere cosiddetto africano (Kezich, Levantesi 2003: 159, 176).

\section{L'architettura di "Una giornata particolare"}

Lo spazio urbano per Scola non è quindi una semplice scenografia, è uno spazio scenico complesso scelto in funzione delle strutture psicologiche dei personaggi. Nel caso di Una giornata particolare (1974) possiamo proprio individuare una stretta relazione tra il luogo selezionato come ambientazione del film e la psicologia dei personaggi. Il film nasce dalla fusione di due temi che riguardano la discriminazione: la condizione della donna e quella omosessuale in un preciso periodo storico, durante il fascismo. È la vicenda di due persone sole, Antonietta e Gabriele, interpretate da Sophia Loren e Marcello Mastroianni. Una casalinga e un annunciatore radiofonico che si ritrovano insieme proprio perché sono immersi nelle rispettive solitudini. I protagonisti si muovono in un palazzone romano "il grattacielo di Roma" 10 che è anche un piccolo paese. Un microcosmo in un grande conglomerato urbano. Nella struttura risiedeva infatti la piccola borghesia fascista rappresentata perlopiù dagli impiegati dei ministeri. I due protagonisti vivono in due appartamenti distinti che si trovano uno di fronte all'altro ma non si sono mai visti prima.

È proprio lo scenografo Luciano Ricceri, nella ricerca della location per il film, a trovare Palazzo Federici nella parte di nord di Roma, vicino a Villa Torlonia che era stata la residenza di Benito Mussolini ${ }^{11}$. Il palazzo, collocato in Viale XXI Aprile ${ }^{12}$ nella zona di Piazza Bologna, è un complesso architettonico disegnato dall'architetto strutturalista Mario De Renzi tra il 1931 e il 1933 e viene ultimato nel 1937, nella scia del piano regolatore dell'epoca. La costruzione risponde ad un modello architettonico, tipico dell'epoca fascista negli anni Trenta, un esempio palese

[...] di crescita urbana basato sul grande intervento privato ai confini della città edificata:
un modello questo messo a punto da [Marcello] Piacentini [il maggior architetto del
ventennio fascista] sulla base delle tipologie urbane tedesche, pubblicizzate in quegli anni
dalla rivista "Architettura e Arti Decorative. [...] La costruzione di queste "case
convenzionate" rientra [quindi] nelle iniziative che il Governatorato di Roma prende per
far fronte alla enorme richiesta di alloggi in seguito al decreto sullo sblocco dei fitti del
1928. Tra le imprese che contribuiscono all'urbanizzazione di interi quartieri vi è anche
l'impresa Federici [da qui il nome del palazzo], per la quale viene progettata questa

\footnotetext{
${ }^{10}$ Lo definisce il questo modo lo scenografo Luciano Ricceri nella sua testimonianza nel volume curato da Tullio Kezich e Alessandra Levantesi. «[...] E io ho trovato questo palazzo Federici, lo chiamavano il "Grattacielo di Roma" perché quando fu costruito non ce ne erano di edifici così alti, una decina di piani più le altane; ed è uno dei principali monumenti dell'edilizia fascista» Cfr. Kezich, Levantesi 2003: 159.

11 Maurizio Costanzo rammenta invece che: «[...] chiacchierando con Ettore, gli suggerii di far fare un sopralluogo a Palazzo Federici, in Viale Ventuno Aprile a Roma. [...]». Cfr. Lettera di Maurizio Costanzo a Tullio Kezich, in Kezich, Levantesi 2003: 176.

${ }^{12}$ Accanto alla Caserma Piave Realizzata da Arnaldo Foschini nel 1914 come l'ingresso della città Universitaria in Piazzale Aldo Moro sotto la supervisione di Marcello Piacentini. Cfr. Carrera et al. 2013: 119.
} 
complessa tipologia abitativa, concepita sulla grande dimensione: sorge su un'area coperta di $5800 \mathrm{mq}$ e comprende 442 appartamenti, 70 negozi, autorimesse ed un cinematografo per 1600 posti [diventato un supermercato nel 1968]. (Neri 1992: 38-42)

Un luogo ideale per descrivere un palazzo "alveare", con una struttura squadrata da piccolo borgo fortificato al cui interno si trovava un cortile centrale con una fontana. Tutta la struttura e l'insieme dei livelli dello spazio si presenta come un piccolo villaggio, il che può essere utile a dimostrare in pieno l'aspetto opprimente del fascismo, soprattutto durante la visita di Hitler a Roma, durante la famosa "giornata particolare" di venerdì 6 maggio del 1938 (Bertini, 1996: 206-207); una giornata che lo stesso Scola ha vissuto personalmente all'età di sei anni con il nonno ${ }^{13}$, il padre e il fratello (Kezich, Levantesi 2003: 145) ${ }^{14}$.

Il film si apre e si chiude proprio con la descrizione della struttura di Palazzo Federici per sottolineare il «clima retorico e soffocante» del periodo fascista in cui erano immersi i due protagonisti (Kezich, Levantesi, 2003: 149).

L'inizio del film, dopo i titoli di testa e la lunga sequenza con il documentario del Luce che illustra l'arrivo di Hitler a Roma, è costituito da due estesi piani sequenza della durata complessiva di 4' 48', che vengono ideati da Scola per far entrare meglio lo spettatore nel clima autoritario di quegli anni.

I primi 14 minuti del film sono così suddivisi in 4 parti: 1) titoli della durata di 1'10',; 2) documentario del Luce con la voce stentorea di Guido Notari, dur. 5'56"; 3 ) primo piano sequenza con la descrizione del cortile e la struttura di Palazzo Federici, dur. 1'15'; 4) secondo piano sequenza con la Mdp che scruta i palazzi, le finestre, le scale e dopo entra senza stacchi - dentro casa di Antonietta, dur. 3' 33'; questo pezzo è interamente girato in studio dove è stato ricostruito l'appartamento dei protagonisti e parte del cortile interno.

Scola descrive così, con un lungo movimento di carrello e lunghe panoramiche - senza audio, se non per la presenza dei rumori d'ambiente - prima la struttura del Palazzo Federici e poi l'appartamento dove vive la protagonista Antonietta/Sophia Loren. La rappresentazione minuziosa delle linee prospettiche di Palazzo Federici è realizzata da Scola proprio per identificare bene che ci troviamo dentro un "borgo urbano", un piccolo paese dentro la città.

Si passa quindi dal bianco e nero del documentario dell'istituto Luce ad un film a colori "slavato" con una dominante marrone che ci offre il complesso architettonico di Palazzo Federici.

Lo spostamento dal bianco e nero del documentario al colore del racconto finzionale è anche evidenziato dal passaggio sonoro della voce stentorea del celebre conduttore radiofonico di quegli anni, Guido Notari (che viene citato da Gabriele/Mastroianni mentre parla con Antonietta/Loren del suo lavoro di conduttore radiofonico), al silenzio di un primo mattino all'interno del condominio romano di Viale XXI Aprile.

Originariamente, Scola voleva fare un film interamente in bianco e nero, ma poi, convinto dal produttore Ponti e dai collaboratori, opta per un film a colori, usando però colori desaturati. In vari momenti di lavoro si trovano diverse soluzioni tecniche sia in fase di

\footnotetext{
${ }^{13}$ Scola aveva sei anni il 6 maggio 1938. Era nato il 10 maggio 1931.

${ }^{14}$ Ettore Scola lo ricorda: «Venerdì 6 maggio 1938. Quella "giornata particolare” me la ricordo bene perché l’ho vissuta. Siamo andati con mio nonno, Pietro, mio padre e mio fratello, Pietro anche lui, a via dell'Impero, alle quattro di mattina. Con pane e frittata, che però alle otto avevamo già mangiato. Eravamo li schierati e ho il ricordo di questo Hitler rosso, di capelli e baffetti [...]» Cfr. in Kezich, Levantesi 2003: 145.
} 
costruzione della scenografia e degli arredi che in fase di ricerca dei costumi, ma soprattutto si realizzano vari espedienti tecnici in ambito fotografico. Pasqualino De Santis, il direttore della fotografia del film (già premio Oscar nel 1969 per la migliore fotografia nel film di Franco Zeffirelli, Romeo e Giulietta), sperimenta un particolare procedimento di stampa del negativo con una specifica "flashatura" che toglie una certa gamma di colori alla versione finale della pellicola. Una desaturazione e decolorazione realizzata in parte nei laboratori della Technicolor per ottenere le tinte slavate che servivano al film di Scola. I colori utilizzati erano perlopiù bianco, nero, verde e rosso e per questo motivo anche la descrizione di Palazzo Federici con quel lungo movimento di macchina ottiene un tono fotografico così particolare e pienamente funzionale ai temi del film. Come ricorda Pasqualino De Santis

\begin{abstract}
la desaturazione [...] non è solo chimica, non è tutto merito della stampa Technicolor. Quelle tinte slavate dipendevano in primo luogo dalla scelta degli ambienti, dei costumi e delle stesse fonti luminose. Le lampadine, ad esempio, dovevano essere arancioni per poi risultare bianche sullo schermo. Il rischio era che venissero "sfondate", troppo luminose, mentre allora la luce era misera, molto fioca. Inoltre c'era un filtro davanti all'obiettivo e poi naturalmente si correggeva la stampa. Gli unici colori del film erano bianco, nero, verde e rosso per questo le immagini hanno quel tono così particolare. Il costumista impostò tutto il suo lavoro in funzione di questi quattro colori. (Kezich, Levantesi 2003: $163,164)$
\end{abstract}

Quindi, grazie alla scelta dei luoghi, ai colori utilizzati e ai piani sequenza introduttivi del film di Scola, che ci descrivono nel dettaglio la struttura architettonica del Palazzo Federici, possiamo ribadire quanto sia determinante il rapporto tra cinema, racconto filmico e architettura.

La narrazione cinematografica si sviluppa perlopiù in rapporto al paesaggio, al movimento degli attori dentro la città, movimenti che corrispondono poi a degli spazi interiori dello spettatore che guarda. In particolare il cinema di Scola ${ }^{15}$ a Roma si muove con sicurezza descrivendo bene psicologie umane e linee prospettiche dello spazio urbano. Il racconto di Una giornata particolare ha quindi «la capacità di rendere gli affetti e di toccarci [soprattutto] nel profondo».

Questo film, inoltre, ci restituisce una performance straordinaria dei due attori che, unita ai movimenti dell'architettura strutturalista presente sullo sfondo, provoca - come suggerisce giustamente Giuliana Bruno - la creazione di «un [incancellabile e personale] archivio delle immagini emozionali» che rimane in maniera indelebile all'interno di ogni spettatore (Bruno 2015: 11-23). L'efficacia narrativa e cinematografica di Antonietta e Gabriele è dovuta anche grazie alle architetture sullo sfondo dello spazio urbano rappresentato da Palazzo Federici in Viale XXI Aprile a Roma.

\title{
3. L'architettura della lingua
}

Come si è detto nel paragrafo precedente, Una giornata particolare è un film particolarmente attento alla dimensione architettonica in correlazione alla psicologia dei

\footnotetext{
${ }^{15}$ Come quello, ad esempio, di Michelangelo Antonioni, forse in maniera più particolare e controversa rispetto a Scola; si pensi ad esempio solo all'isola Tiberina del rivoluzionario L'avventura del 1960 e da La Notte e L'eclisse, nella trilogia dell'incomunicabilità (Bernardi 2002).
} 
personaggi. Tale correlazione si realizza anche in una dimensione linguistica, per cui alla conformazione dello spazio rappresentato corrisponde una particolare architettura linguistica.

I film di Ettore Scola regista, dal primo Se permettete parliamo di donne (1964) fino all'esperimento della serie di micro racconti urbani di Gente di Roma (2003) dedicato alla sua città d'adozione, sono esempi molto chiari di come la lingua - quindi le scelte linguistiche tra le varietà del diasistema italiano, tra i dialetti e le altre lingue presenti nel territorio - giochi un ruolo fondamentale. Ciò avviene con più decisione e precisione, e sicuramente con un profondo piacere personale, quando Scola si concentra sulla rappresentazione della realtà romana, soffermandosi con intelligenza e sensibilità sulla rappresentazione della situazione sociolinguistica dello spazio urbano e sulla sua funzione narrativa. Inoltre, Scola riesce a mostrare come tali scelte seguano le esigenze di rappresentazione della realtà e delle dinamiche narrative legate al luogo e al contesto che il film vuole raccontare.

Il cinema di Scola è rivolto proprio all'osservazione, spesso ironica e pungente, delle contraddizioni della società italiana, dei malumori e dei vizi degli italiani, raccontando la storia d'Italia dal punto di osservazione del quotidiano. Sue immagini privilegiate, anche nei tanti documentari da lui realizzati, sono quelle immagini legate agli uomini e alle donne in crisi di identità, quelle varie storie legate al boom economico e ai caotici sviluppi urbanistici, quella trasformazione accelerata delle nostre città, con grandi masse di popolazione che si spostano dai campi verso le città e dal sud al nord in cerca di lavoro, ha trasformato la cultura, anche quella popolare, dell'Italia. Questo fenomeno, questo fermento, questa crisi entrano, infatti, anche nello schermo cinematografico degli sceneggiatori e dei registi più sensibili, che proprio come Ettore Scola, vogliono raccontare questa trasformazione che riguarda, è noto, anche i comportamenti linguistici.

Rimandando a un'altra sede ciò che può dirsi riguardo al capolavoro del grottesco (ed è grottesco non solo nelle immagini e nella storia, nei luoghi e nei tanti personaggi rappresentati, ma anche nelle scelte linguistiche) di Brutti, sporchi e cattivi ${ }^{16}$ (1976) o il drammatico e feroce miscuglio tra commedia e noir del Romanzo di un giovane povero (1995), come già mostrato nel paragrafo precedente, ci concentreremo sull'esempio del film Una giornata particolare (1977).

In questo film sul quotidiano al cospetto della storia, secondo la definizione di Carmelo Marabello (2016: 76), appare chiaro come il cinema di Scola sia un cinema di osservazione e che funzioni anche come strumento per raccontare il Paese, cioè come mezzo efficace del regista per studiare e conoscere meglio la realtà e, eventualmente, per contribuire a migliorarla.

Una giornata particolare comincia con la presentazione della messa in scena del regime fascista pomposo, monumentale, autocelebrativo e retorico, utilizzando le immagini d'epoca tratte da un documentario Luce, il quale riprende e commenta le scene dell'adunata organizzata in occasione della visita di Hitler a Roma. Subito dopo le immagini d'epoca, Scola ci porta all'interno di un fascismo del quotidiano, più familiare e in qualche modo intimo.

Il regista va così a spostare il velo e a scoprire le fragilità di un sistema sociale e familiare tutto basato su una struttura superficiale, ambigua e traballante. Così, l'ambientazione nel fittissimo condominio di Palazzo Federici è funzionale a questo modo di

\footnotetext{
${ }^{16}$ Per un'analisi della lingua di Brutti, sporchi e cattivi, si veda Capotosto 2016.
} 
procedere che conduce dalla visione esterna a una osservazione interna, intima e partecipata. La messa in scena ci conduce dal fatto storico al fatto privato, seguendo un processo di sgretolamento della storia che ci introduce alla microstoria consumata in una giornata che si rivelerà particolare sotto diversi punti di vista (cfr. anche Uva 2016: 204). La narrazione cinematografica, dalla «tessitura post-neorealista e post-viscontiana» (Marabello 2016: 11), si sposta poi su un altro livello, andando a interessare nuovamente la macrostoria sociale italiana, cioè la questione della posizione della donna nella società e la discriminazione nei confronti delle persone omosessuali. Difatti, la riflessione sul passato consente di operare un'analisi più accurata del presente e verificare la nostra propensione a favorire i mutamenti nelle strutture sociali italiane.

Antonietta è una sposa devota e rassegnata, dedita alla cura della casa, del marito e dei loro sei figli. Affascinata, per noia e consuetudine sociale, più che per reale convinzione, dalla figura del Duce, conduce un'esistenza senza interessi e senza grandi passioni che non siano legate alla numerosa famiglia o alla devozione nei confronti di Mussolini. Antonietta cura con molta attenzione la sua passione per il duce, ne ha fatto un ritratto realizzando un mosaico di bottoni e ne conserva le foto in un album, sul quale riporta anche alcune sue frasi. Gabriele è un radiocronista omosessuale che è stato appena licenziato dalla EIR, l'Ente Italiano per le Audizioni Radiofoniche, proprio a causa delle sue preferenze sessuali. Il film racconta l'incontro di due solitudini emarginate, di due membri della società che sono considerati, in maniera e per ragioni diverse, inferiori: Antonietta è inferiore in quanto donna, ma inclusa nella società in quanto moglie e madre; Gabriele è inferiore in quanto non ricopre nessun ruolo sociale utile, non è infatti né padre, né uomo, né soldato. Mentre Gabriele è un escluso, Antonietta ha una sua collocazione attraverso la quale le è assicurata un'identità di facciata riconoscibile come utile.

Come abbiamo ricordato all'inizio, il film si apre proprio sulla Roma fascista, e attraverso le immagini della città che celebra il dittatore nazista in visita: la lingua del documentario è carica della retorica di regime e ci prepara a un certo fastidio nei confronti di quella pomposità celebrativa per Hitler, accompagnata dalle note e dai versi dell'inno trionfale fascista Giovinezza. Dopo il tripudio di immagini e di folla acclamante, di svastiche e saluti romani, torna il silenzio della mattina all'interno di uno degli appartamenti del borgo metropolitano di palazzo Federici.

Antonietta è la prima a svegliarsi e piano piano sveglia il resto della famiglia.

Il marito Emanuele (John Vernon), uomo volgare e antipatico, mette immediatamente in evidenza il rapporto sbilanciato tra lui e sua moglie: si lamenta infatti per essere stato svegliato borbottando «devi rompere i cojoni de matina». I toni sono della commedia amara, la scena del risveglio dei figli è un alternarsi di momenti ironici e momenti amari. La parte meno ironica è quella del marito, ridicolizzato semmai per la continua ricerca di affermazione della propria virilità, con il suo comportamento volgare e i tanti tradimenti di cui Antonietta è a conoscenza - tradimenti che non sarebbero stati percepiti tanto offensivi e gravi finché si fossero limitati alla frequentazione dei bordelli cittadini, ma diventano più gravi quando il tradimento comporta una relazione anche affettiva con una maestra, cioè con una donna istruita. Questo fatto fa sentire Antonietta ancora una volta inadeguate e perdente. La scena, con il già citato piano sequenza che mostra un appartamento umile e sovraffollato di figli, si svolge in una lingua parlata molto realistica, una lingua mista tra il napoletano accennato di Antonietta e il romanesco del resto della famiglia. 
Alcune battute ironiche fanno da contraltare alle immagini retoriche appena viste e alleggeriscono il clima della scena: Maria Luisa (Alessandra Mussolini), la figlia più grande, sempre in ritardo «tu sei capace di far aspettare pure Hitler» e più interessata a farsi bella che all'adunata in sé, «vai all'adunata/ o alla festa da ballo?», e Umberto (Vittorio Guerrieri), il figlio maggiore, che cerca di sembrare più adulto disegnandosi i baffi allo specchio. Con quest'ultimo, Antonietta scambierà alcune battute in un divertente siparietto sulla masturbazione. Nel rifare il letto, infatti, Antonietta trova il ritaglio di una rivista con le foto di alcune donne nuda:

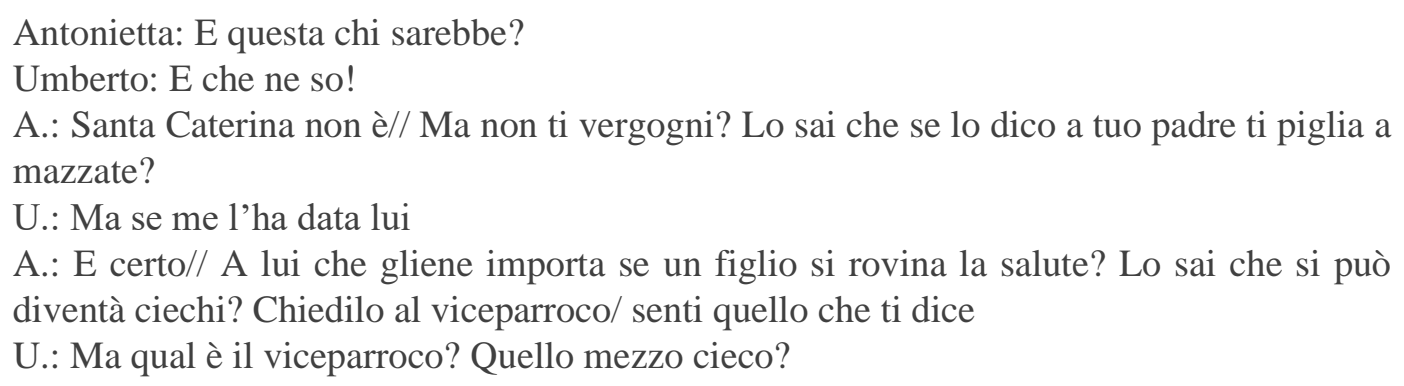

Mentre tutti si vestono, inoltre, uno dei figli nomina il pon-pon del cappello da giovane balilla e Emanuele lo riprende per aver usato una parola straniera: «Non si dice ponpon/ è parola straniera! Chiamalo fiocco/ nappa/ non so/ chiamalo ponpono».

Ettore Scola e Ruggero Maccari e Maurizio Costanzo, gli altri sceneggiatori del film, fanno varie volte riferimento alla politica linguistica autarchica del fascismo. In particolare, qui viene messa in evidenza l'avversione nei confronti delle lingue straniere e nei confronti dell'uso dell'allocutivo di cortesia lei in luogo della forma voi, ritenuta più italiana e più virile $^{17}$.

La lingua di Antonietta è incerta e ha segni di italiano regionale campano e di italiano popolare (es. «non me l'ho dimenticato») mentre Emanuele, come già detto, è volgare, aggressivo e infarcito di retorica machista fascista («Sbrigateve 'n po' morbidoni»). L'incontro tra Antonietta e Gabriele avviene grazie alla fuga di Rosmunda, la merla parlante che vola dall'appartamento di Antonietta fino ad un cornicione vicino alla finestra di Gabriele.

L'inizio è timido e imbarazzato, poi i due si trovano a proprio agio e si mostrano l'un all'altra per come realmente sono. Le lunghe chiacchierate li portano sul terrazzo condominiale, e tra file di lenzuola bianche agitate dal vento, Antonietta rivela di essere attratta dall'uomo: gli si avvicina, e mentre prima gli chiede di andarsene, poiché non riesce a gestire queste nuove emozioni, poi gli afferra le mani, lo accarezza, lo bacia. Gabriele non reagisce, è impassibile. Poi confessa:

Gabriele: C'è una frase nel tuo albo/ l'uomo deve essere marito/ padre e soldato// Io non sono/ né marito/ né padre e né soldato//

Antonietta: Che vuol dire?

G.: Non mi hanno mandato via dalla radio per la mia voce// Disfattista/ inutile e con tendenze depravate/ così hanno detto//

\footnotetext{
${ }^{17}$ Come sappiamo, la disposizione fascista in favore del voi ebbe scarsa efficacia, non solo perché fu varata solo nel 1938, ma anche perché il lei era molto diffuso, mentre il voi era concentrato nel Mezzogiorno (cfr. Serianni 2000: 7).
} 


\section{A.: Non capisco// \\ G.: Hai capito benissimo// È così//}

Antonietta reagisce male. È delusa e arrabbiata con sé stessa. Si è esposta, ha osato, e dall'altra parte non ha trovato ciò che si aspettava, ciò che sperava di trovare. Colpisce Gabriele con un violento schiaffo e scappa. L'uomo la rincorre, la afferra, la palpeggia, la insulta, si comporta come si sarebbe comportato un uomo virile fascista, conquistatore di corpi femminili, ma lui non è che un «frocio», un «ricchione». Gabriele lo grida: «Io non sono quel maschione virile che speravi// Io sono un frocio// Frocio!», aggiungendo poi, con ancora maggior ardore: «Così lo sapranno tutti/ finalmente/ che l'inquilino del sesto piano è ricchione! È finocchio!». È questa la scena madre del film, quella delle rivelazioni.

Dopo questo scontro, Antonietta e Gabriele diventano intimi confidenti: «Pure io tante volte mi sento umiliata/ considerata meno di zero// Mio marito con me non parla/ ordina// Di giorno/ e di notte», rivela finalmente Antonietta dando sfogo alla sua malinconia».

Alla fine della giornata, le due solitudini tornano ad essere tali, entrambi tornano in qualche modo da Mussolini. Lo stesso Ettore Scola descrive Una giornata particolare come «la storia di due isolati che incontrandosi e riconoscendosi come esclusi riescono in poche ore dal ruolo di cittadini di seconda categoria nel quale sono confinati, e a diventare protagonisti di loro stessi, dei loro impulsi, del loro quotidiano» (Scola 1977: 135).

In una delle sue ultime battute, Gabriele, congedandosi da Antonietta, afferma: «Essere come sono io non significa non poter fare l'amore con una donna// È diverso// È stato bello/ ma non cambia niente». Difatti, l'esperienza di questa giornata non ha cambiato la natura sessuale di Gabriele; il rapporto sessuale è servito più ad Antonietta come esperienza di rottura per recuperare la propria natura femminile, non per compiere un'operazione di salvazione della virilità di Gabriele, ma per riaffermare il proprio essere donna capace di compiere le proprie scelte.

Qualcosa è cambiato nelle vite di questi due individui che ora si sentono meno soli, nella complicità, nell'affettuosa intimità che si è realizzata anche nel sesso, come momento di intima sincerità. E ciò avviene anche nella lingua, nel sistema di segni in cui i due protagonisti sono imprigionati, seppur diversi sono i gradi di consapevolezza. Difatti, solo attraverso il rovesciamento dei significati simbolici assegnati alle etichette sociali, i personaggi riescono a prendere una vera coscienza di sé e di accettare di poter vivere una condizione sociale diversa da quella in cui si vuole essere imprigionati.

Questa è l'architettura della lingua che viene rappresentata nel film. Come strumento sociale, infatti, la lingua funge anche da difesa. Tale funzione si attenua però lentamente, man mano che la comunicazione diventa una questione intima e privata, andando a intaccare la struttura di una società piena di contraddizioni. I rimandi alla politica linguistica del fascismo e a quella lingua che deve essere prima di tutto virile, servono proprio per rappresentare la superficialità della struttura dei rapporti tra gli individui. Le incursioni macchiettistiche della portinaia pettegola sono usate per ribilanciare il rapporto di intimità tra Antonietta e Gabriele, e simboleggiano le ingerenze della dittatura nella vita privata e nella sfera intima delle persone. 


\section{Osservazioni conclusive}

Abbiamo visto come una visione deformata in direzione espressionista caratterizza la prima parte del film e investe non soltanto l'architettura di Palazzo Federici, ma anche le strutture portanti della lingua. Le linee strutturali dell'architettura convergono tutte verso il fuoco del potere, rappresentato dalla portinaia pettegola, che svolge il ruolo di controllore sociale. Allo stesso modo, le linee strutturali del rigido sistema sociolinguistico ci appaiono incombere sulla quotidianità dei suoi abitanti, e in particolare sulle vite di Antonietta e Gabriele.

Questa prima parte del film, infatti, è caratterizzata da un uso espressionista del romanesco, al quale i riferimenti alla politica linguistica del fascismo aggiungono una patina grottesca. La dimensione carceraria foucaultiana, come la definisce Christian Uva (2015: 285), riguarda anche la struttura della lingua, in cui si trovano intrappolati i personaggi, almeno fino alla scena del bacio sulla terrazza. Questa scena, come già detto, è cruciale nel rappresentare il ribaltamento dei rapporti tra pubblico e privato. Difatti, l'unione tra Antonietta e Gabriele si realizza dopo aver forzato tutte le regole di conformità sociale, passando dall'intimità ancora superficiale creatasi quando i due personaggi entrano una nell'appartamento dell'altro e viceversa, fino al crescendo, anche nel senso dello spazio físico rappresentato dalle scale che portano verso la libertà, nella terrazza ${ }^{18}$. Qui sulla terrazza, unico territorio che sembrerebbe libero dagli sguardi della portiera, si sgretolano, seppure in modo traumatico, le categorie del conformismo fascista e i due personaggi possono rivelarsi per le persone che sono. Immediatamente dopo, arriva la paura di trovarsi senza sostegno e Antonietta scappa nuovamente a trovare rifugio nel labirinto del condominio. Come sappiamo, però, il cambiamento è compiuto, le parole tra i due sono sempre meno perché anche l'architettura della lingua è spezzata. Pian piano tutti i condomini tornano dalla parata, come per tentare di compiere la ristrutturazione di un impianto architettonico sociale e linguistico che ha ormai mostrato tutta la sua fragilità.

\section{Bibliografia}

ASC Incontri, anno VII, n.2, Intervista a Ettore Scola, pp. 18-22.

ASC Incontri, anno VII, n.2, Intervista a Luciano Ricieri, pp. 13-14.

Bernardi Sandro, 2002, Il paesaggio nel cinema italiano, Venezia, Marsilio.

Bertini Antonio (a cura di), 1996, Ettore Scola. Il cinema e io, Roma, Officina edizioni, pp. 206-207.

Bruno Giuliana, 2009, Pubbliche intimità. Architettura e arti visive (2007), Milano, Bruno Mondadori.

Bruno Giuliana, 2015, Atlante delle emozioni. In viaggio tra arte, architettura e cinema (2002), Johan \& Levi.

Bruscolini Elisabetta (a cura di), 2002, Rome in cinema between reality and fiction, Venezia, Marsilio.

\footnotetext{
${ }^{18}$ Le scene sulla terrazza sono state girate perlopiù nel palazzo-ospedale "George Eastman", sempre nella zona nord di Roma in Viale Regina Elena 287, poco prima della città universitaria realizzata negli anni Trenta sotto la supervisione dell'architetto Marcello Piacentini (Carrera 2013).
} 
Capotosto Silvia, 2016, I dialetti e il continuum in Brutti, sporchi e cattivi di Ettore Scola (1976), in Marco Gargiulo (a cura di), Lingue e linguaggi del cinema in Italia, Roma, Aracne, pp. 173-197.

Carrera Manuel, D’Angelo Giulia, D’Ecclesia Silvia, Foscolo Giulia, Gandolfi Riccardo, (a cura di), 2013, Sapienza razionalista. L'architettura degli anni '30 nella città universitaria, Roma, Edizioni Nuova Cultura.

Costa Antonio, 2002, Il cinema e le arti visive, Torino, Einaudi.

Dionisi Marco, De Pascalis Nevio (a cura di), 2016, Piacere Ettore Scola, Roma, Edizione Sabine, pp. 274-277.

Kezich Tullio, Levantesi Alessandra, 2003, Una giornata particolare, un film di Ettore Scola. Incontrarsi e dirsi addio nella Roma del '38, Torino, Lindau.

Marabello Carmelo, 2016, Quotidiano, in De Gaetano R. (a cura di) Lessico del cinema italiano. Forme di rappresentazione e forme di vita, vol. III, Milano-Udine, Mimesis, pp. 7-92.

Iarussi Oscar, 2017, Andare per i luoghi del cinema, Bologna, Il Mulino.

Insolera Italo, 1971, Roma moderna. Un secolo di storia urbanistica, Torino, Einaudi.

Neri Maria Luisa, 1992, Mario De Renzi. L'architettura come mestiere 1897-1967, Roma, Gangemi.

Nicoloso Paolo, 2011, Mussolini architetto. Propaganda e paesaggio urbano nell'Italia fascista, Torino, Einaudi.

Prina Vittorio, 2009, Cinema, architettura, composizione, Rimini, Maggioli.

Siciliano Enzo (a cura di), 2001, C'eravamo tanto amati di Ettore Scola. Storie di italiani. Storia d'Italia, Torino, Lindau.

Scola Ettore, 1977, Una giornata particolare del '38, in Scola E., Maccari R., Costanzo M., Una giornata particolare: soggetto e sceneggiatura, Milano, Longanesi.

Serianni Luca, 2000, Risposta al quesito del signor Guido Iazzetta di Milano sull'uso degli allocutivi, «La Crusca per voi», 20, p. 7.

Uva Christian, 2015, Un borgo nella metropoli. Ettore Scola a Palazzo Federici, «The Italianist», 35. 2, pp. 284-290.

Uva Christian, 2016, Storia, in De Gaetano R. (a cura di) Lessico del cinema italiano. Forme di rappresentazione e forme di vita, vol. III, Milano-Udine, Mimesis, pp. 163-227. 